\title{
Decay of Strong Solutions for 4D Navier-Stokes Equations Posed on Lipschitz Domains
}

\author{
N. A. Larkin \\ Departamento de Matemática, Universidade Estadual de Maringá, Av. Colombo 5790, Agência UEM, 87020-900, Maringá, PR, Brazil \\ Correspondence should be addressed to N. A. Larkin; nlarkine@uem.br
}

Received 14 August 2018; Accepted 27 November 2018; Published 13 December 2018

Academic Editor: Sergey Shmarev

Copyright (c) 2018 N. A. Larkin. This is an open access article distributed under the Creative Commons Attribution License, which permits unrestricted use, distribution, and reproduction in any medium, provided the original work is properly cited.

Initial-boundary value problems for 4D Navier-Stokes equations posed on bounded and unbounded 4D parallelepipeds were considered. The existence and uniqueness of regular global solutions on bounded parallelepipeds and their exponential decay as well as the existence, uniqueness, and exponential decay of strong solutions on an unbounded parallelepiped have been established provided that initial data and domains satisfy some special conditions.

\section{Introduction}

This work concerns the existence and uniqueness of global strong solutions and sharp decay estimates of solutions to initial-boundary value problems for the 4D Navier-Stokes equations:

$$
\begin{aligned}
u_{t}+(u \cdot \nabla) u & =v \Delta u-\nabla p, \quad \text { in } \Omega \times(0, t), \\
\nabla \cdot u & =0 \quad \text { in } \Omega, \\
\left.u\right|_{\partial \Omega} & =0 \\
u(x, 0) & =u_{0}(x),
\end{aligned}
$$

where $\Omega$ is either a bounded or an unbounded parallelepiped in $\mathbb{R}^{4}$ with the homogeneous Dirichlet condition on the boundary of $\Omega$.

The question of decay of the energy for weak solutions had been stated by J. Leray in [1] and attracts till now attention of many pure and applied mathematicians [2-9]. In all of these papers, the decay rate of $\|u\|(t)_{L^{2}(\Omega)}$ was controlled by the first eigenvalue of the operator $A=-P \Delta$, where $P$ is the projection operator on the solenoidal subspace of $L^{2}(\Omega)$. Obviously, this approach does not work in unbounded domains; see $[6,7,9]$.

It is well known that solutions of the 2D Navier-Stokes equations posed on smooth bounded domains with the Dirichlet boundary conditions are globally regular $[4,6-9]$. On the other hand, the question of regularity for $3 \mathrm{D}$ and $4 \mathrm{D}$
NSE with arbitrary initial data is till now an open problem even for smooth domains; see $[6,7,9]$. Small initial data help to solve this problem $[6,7,9]$ as well as the so-called "thin" domains when some size of a domain is small [10, 11]. The question of regularity becomes more difficult while a domain is Lipschitzian $[10,12,13]$.

In $[6,7,9,14]$, it has been proved that for 3D Lipschitz domains, bounded and unbounded and small initial data there exists a unique global strong solution

$$
u, u_{t} \in L^{\infty}\left(0, \infty ; L^{2}(\Omega)\right) \cap L^{2}\left(0, \infty ; H^{1}(\Omega)\right),
$$

but it was not clear whether

$$
u \in L^{\infty}\left(0, \infty ; H^{2}(\Omega)\right)
$$

at least for bounded Lipschitz domains.

Our goal here is making some geometrical restrictions, to prove the existence and uniqueness of strong global solutions in 4D Lipschitz domains for arbitrary regular initial data as well as exponential decay of solutions.

In this work, making use of ideas of [15], we have established that $u \in W^{2,4 / 3}(\Omega)$ for a $4 \mathrm{D}$ bounded parallelepiped. The following inequality holds:

$$
\|u\|_{H_{0}^{1}(\Omega)}^{2}(t)+\left\|u_{t}\right\|_{L^{2}(\Omega)}^{2}(t) \leq C\left\|u_{0}\right\|_{H^{2}(\Omega)}^{2} \exp (-v \chi t),
$$

where $\chi>0$. 
Our paper has the following structure: Section 1 is Introduction. Section 2 contains notations and auxiliary facts. In Section 3, existence, uniqueness, and decay of global strong solutions on a bounded 4D parallelepiped have been established. In Section 4, the existence, uniqueness, and decay of regular solutions on bounded $4 \mathrm{D}$ parallelepipeds and strong solutions on $4 \mathrm{D}$ unbounded parallelepipeds have been demonstrated.

\section{Notations and Auxiliary Facts}

Let $x=\left(x_{1}, x_{2}, x_{3}, x_{4}\right)$ and $\Omega$ be a domain in $\mathbb{R}^{4}$. Define as in [9], p.2-4

$$
\begin{aligned}
D_{i} & =\frac{\partial}{\partial x_{i}}, \quad i=1,2,3,4 ; \\
D^{\alpha} & =\prod_{i=1}^{4} D_{i}^{\alpha_{i}}, \quad \alpha=\sum_{i=1}^{4} \alpha_{i}, \\
u & =\left(u_{1}, u_{2}, u_{3}, u_{4}\right) ; \\
u_{t} & =\left(u_{1 t}, u_{2 t}, u_{3 t}, u_{4 t}\right) ; \\
D_{i} u & =\left(D_{i} u_{1}, D_{i} u_{2}, D_{i} u_{3}, D_{i} u_{4}\right) .
\end{aligned}
$$

We denote for scalar functions $f(x)$ the Banach space $L^{p}(\Omega), 1<p<+\infty$ with the norm

$$
\begin{aligned}
\|f\|_{L^{p}(\Omega)}^{p} & =\int_{\Omega}|f|^{p} d x, \quad p \in(1,+\infty), \\
\|f\|_{L^{\infty}(\Omega)} & =\underset{\Omega}{\operatorname{ess} \sup }|f(x)| .
\end{aligned}
$$

$$
\mathscr{V}=\{u \in \mathscr{D}(\Omega), \nabla \cdot u=0\}, \quad V=\text { the closure of } \mathscr{V} \text { in } H_{0}^{1}(\Omega), H=\text { the closure of } \mathscr{V} \text { in } L^{2}(\Omega)
$$

The space $H$ is equipped with the natural $L^{2}$ inner product. The space $V$ will be equipped with the scalar product

$$
((u, v))=\sum_{i=1}^{4}\left(D_{i} u, D_{i} v\right)
$$

when $\Omega$ is bounded. If $\Omega$ is unbounded, we define the inner product as the sum of the inner products as follows:

$$
[[u, v]]=(u, v)+((u, v))
$$

We use the usual notations of Sobolev spaces $W^{k, p}, L^{p}$, and $H^{k}$ for vector functions and the following notations for the norms:
For $p=2, L^{2}(\Omega)$ is a Hilbert space with the scalar product

$$
\begin{aligned}
& (u, v)=\int_{\Omega} u(x) v(x) d x \text { and the norm } \\
& \|u\|^{2}=\int_{\Omega}|u(x)|^{2} d x .
\end{aligned}
$$

When $p=2, W^{m, 2}(\Omega)=H^{m}(\Omega)$ is a Hilbert space with the following scalar product and the norm:

$$
\begin{aligned}
((u, v))_{H^{m}(\Omega)} & =\sum_{|j| \leq m}\left(D^{j} u, D^{j} v\right), \\
\|u\|_{H^{m}(\Omega)}^{2} & =\sum_{|j| \leq m}\left\|D^{j} u\right\|^{2} .
\end{aligned}
$$

Let $\mathscr{D}(\Omega)$ or $\mathscr{D}(\bar{\Omega})$ be the space of $C^{\infty}$ functions with compact support in $\Omega$ or $\bar{\Omega}$. The closure of $C^{\infty}$ functions in $W^{m, p}(\Omega)$ is denoted by $W_{0}^{m, p}(\Omega)$ and $\left(H_{0}^{m}(\Omega)\right.$ when $\left.p=2\right)$.

Define the auxiliary spaces which are projections for the solenoidal vector functions,

(i) For vector functions $u(x)=\left(u_{1}(x), u_{2}(x), u_{3}(x)\right.$, $\left.u_{4}(x)\right)$,

$$
\begin{aligned}
\|u\|_{L^{p}(\Omega)}^{p} & =\int_{\Omega}\left(\sum_{i=1}^{4}\left|u_{i}\right|^{p}\right) d x, \\
\|u\|_{W^{k, p}(\Omega)} & =\sum_{0 \leq|\alpha| \leq k} \sum_{i=1}^{4}\left\|D^{\alpha} u_{i}\right\|_{L^{p}(\Omega)}, \quad p \in(1,+\infty) .
\end{aligned}
$$

The closures of $\mathscr{V}$ in $L^{2}(\Omega)$ and in $H_{0}^{1}(\Omega)$ are the basic spaces in our study. We denote them by $H$ and $V$, respectively.

Remark 1. By definition, $V$ is a proper subspace of $H_{0}^{1}(\Omega)$.

Define the operator

$$
(u \cdot \nabla) u=\sum_{i=1}^{4} u_{i} D_{i} u .
$$


Lemma 2 (the Steklov inequality [16]). Let $v \in H_{0}^{1}(0, L)$. Then

$$
\frac{\pi^{2}}{L^{2}}\|v\|^{2}(t) \leq\left\|v_{x}\right\|^{2}(t) .
$$

Proof. Let $v(t) \in H_{0}^{1}(0, \pi)$, then by the Fourier series,

$$
\int_{0}^{\pi} v_{t}^{2}(t) d t \geq \int_{0}^{\pi} v^{2}(t) d t
$$

Inequality (17) follows by a simple scaling.

The next lemmas will be used in estimates.

Lemma 3 (see: [17] Theorem 7.1, p.14). Let $v \in H_{0}^{1}(\Omega)$, then

$$
\|v\|_{L^{4}(\Omega)} \leq 3\|\nabla v\|_{L^{2}(\Omega)} .
$$

Lemma 4. Let $b(u, v, w)=((u \cdot \nabla) v, w)$, then

$$
\begin{aligned}
|b(u, v, w)| & \leq\|u\|_{L^{4}(\Omega)}\|v\|_{V}\|w\|_{L^{4}(\Omega)} \\
& \leq 3^{2}\|u\|_{V}\|v\|_{V}\|w\|_{V}
\end{aligned}
$$

$\forall u, v, w \in H_{0}^{1}(\Omega)$. If $u \in L^{\infty}(0, \infty ; V)$, then we can define the operator Bu such that $B u$ belongs to $L^{\infty}\left(0, \infty ; V^{\prime}\right)$ and

$$
\begin{aligned}
\langle B u, v\rangle & =b(u, u, v), \\
\|B u\|_{L^{\infty}\left(0, \infty ; V^{\prime}\right)} & \leq 3^{2}\|u\|_{L^{\infty}(0, \infty ; V)}^{2} .
\end{aligned}
$$

\section{Existence Theorems}

Let $\Omega$ be a bounded 4D parallelepiped: $\Omega=\left[x \in \mathbb{R}^{4} ; x_{i} \in\right.$ $\left.\left(0, L_{i}\right) ; L_{i}>0, i=1,2,3,4\right]$ which is a Lipschitz domain. Denote $L=\min \left(L_{1}, L_{2}, L_{3}, L_{4}\right)$. Given $u_{0} \in H$, consider the following problem:

$$
\begin{array}{rlrl}
u_{t}-v \Delta u+\nabla p+(u \cdot \nabla) u=0 & & \text { in } \Omega \times(0, t), \\
\nabla \cdot u=0 & & \text { in } \Omega \times(0, t), \\
u & =0 & \\
& \quad \text { on } \partial \Omega \times(0, t), t>0, \\
u(x, 0) & =u_{0}(x), & \text { in } \Omega
\end{array}
$$

equivalent to the variational problem given by (see [7], [9] Problem 3.2, p. 191.)

$$
\begin{aligned}
u^{\prime}+A u+B u & =0 \quad \text { in }(0, t), t>0 \\
u(0) & =u_{0},
\end{aligned}
$$

where $A u \in V^{\prime}$ such that $\langle A u, v\rangle=v((u, v))$ for all $v \in V$ and $B u \in V^{\prime}$ such that

$$
\langle B u, v\rangle=b(u, u, v) .
$$

Theorem 5. Given $u_{0} \in H^{2}(\Omega) \cap V$ and $\Omega$ such that

$$
\left(\frac{\pi}{L}\right)^{1 / 2} \nu^{7 / 4}-18\left\|u_{0}\right\|^{1 / 4}\left\|u_{t}\right\|(0)^{3 / 4}>0,
$$

there exists a unique strong solution $u(x, t)$ to (22):

$$
\begin{aligned}
& u \in L^{\infty}(0, \infty ; V), \\
& u_{t} \in L^{\infty}(0, \infty ; H) \cap L^{2}(0, \infty ; V)
\end{aligned}
$$

such that for all $\Phi: \mathbb{R}^{4} \longrightarrow \mathbb{R}^{4} \in V,\left.\Phi\right|_{\partial \Omega}=0$ it satisfies the following identity:

$$
\left(u_{t}, \Phi\right)(t)+v\left(\sum_{i=1}^{4} D_{i} u, D_{i} \Phi\right)(t)+b(u, \Phi, u)(t)=0,
$$

$$
t>0 .
$$

Moreover, the following inequalities hold:

$$
\begin{aligned}
\|u\|(t) & \leq\left\|u_{0}\right\| e^{-\chi t}, \\
\left\|u_{t}\right\|(t) & \leq\left\|u_{t}\right\|(0) e^{-(\chi / 2) t}, \\
\mid u \|(t)_{V} & \leq C\left\|u_{0}\right\|\left\|u_{t}\right\|(0) e^{-(3 / 4) \chi t},
\end{aligned}
$$

$$
\text { where } \chi=v \pi^{2} \sum_{i=1}^{4}\left(1 / L_{i}^{2}\right) \text {. }
$$

Proof. The estimates that follow may be established on Galerkin's approximations (see [14], [6] p. 136-140, [7], [9], p. 192-197.).

Estimate $I\left(u \in L^{\infty}(0, \infty ; H) \cap L^{2}(0, \infty ; V)\right)$. Multiply (23) by $u \in V$ to obtain

$$
\left(u_{t}, u\right)(t)+(A u, u)(t)=0 .
$$

It follows from here that

$$
\frac{d}{d t}\|u\|^{2}(t)+2 v\|u\|_{V}^{2}(t)=0 .
$$

Making use of the Steklov inequalities, we get

$$
\|\nabla u\|^{2}(t) \geq \pi^{2} \sum_{i=1}^{4} \frac{1}{L_{i}^{2}}\|u\|^{2}(t) .
$$

Returning to (30), we obtain

$$
\frac{d}{d t}\|u\|^{2}(t)+2 \chi\|u\|^{2}(t) \leq 0
$$

which implies

$$
\|u\|^{2}(t) \leq\left\|u_{0}\right\|^{2} e^{-2 \chi t}, \quad \chi=v \pi^{2} \sum_{i=1}^{4} \frac{1}{L_{i}^{2}} .
$$

This and (30) give

$$
u \in L^{\infty}(0, \infty ; H) \cap L^{2}(0, \infty ; V) .
$$

Estimate II $\left(u_{t} \in L^{\infty}(0, \infty ; H) \cap L^{2}(0, \infty ; V)\right)$. Differentiating (23) and multiplying by $u_{t}$, we get

$$
\frac{d}{d t}\left\|u_{t}\right\|^{2}(t)+2 v\left\|u_{t}\right\|_{V}^{2}(t)+2 b\left(u_{t}, u, u_{t}\right)(t)=0 .
$$


We estimate

$\left|b\left(u_{t}, u, u_{t}\right)(t)\right| \leq\left\|u_{t}\right\|(t)_{L^{4}(\Omega)}\|u\|_{V}(t)\left\|u_{t}\right\|(t)_{L^{4}(\Omega)}$.

By Lemma 3,

$$
2\left|b\left(u_{t}, u, u_{t}\right)(t)\right| \leq 2 \cdot 3^{2}\left\|u_{t}\right\|_{V}^{2}(t)\|u\|_{V}(t)
$$

and (35) becomes

$$
\begin{aligned}
\frac{d}{d t}\left\|u_{t}\right\|^{2}(t)+v\left\|u_{t}\right\|_{V}^{2}(t) \\
\quad+(v-18\|\nabla u\|(t))\left\|\nabla u_{t}\right\|^{2}(t) \leq 0 .
\end{aligned}
$$

Making use of (30), we find

$$
v\|\nabla u\|^{2}(t) \leq\|u\|(t)\left\|u_{t}\right\|(t) .
$$

Substituting this into (38), we get

$$
\begin{aligned}
& \frac{d}{d t}\left\|u_{t}\right\|^{2}(t)+v\left\|u_{t}\right\|_{V}^{2}(t) \\
& \quad+\left(v-\frac{18\|u\|^{1 / 2}(t)\left\|u_{t}\right\|^{1 / 2}(t)}{v^{1 / 2}}\right)\left\|\nabla u_{t}\right\|^{2}(t) \leq 0 .
\end{aligned}
$$

By Lemma 2,

$$
\|u\|(t) \leq \frac{L}{\pi}\|\nabla u\|(t) .
$$

Substituting this into (40) and using (39), we find

$$
\begin{aligned}
& \frac{d}{d t}\left\|u_{t}\right\|^{2}(t)+v\left\|u_{t}\right\|_{V}^{2}(t) \\
& \quad+\left(v-\frac{18 L^{1 / 2}\|\nabla u\|^{1 / 2}(t)\left\|u_{t}\right\|^{1 / 2}(t)}{v^{1 / 2} \pi^{1 / 2}}\right)\left\|\nabla u_{t}\right\|^{2}(t)
\end{aligned}
$$$$
\leq 0 \text {. }
$$

Again by (39),

$$
\begin{aligned}
& \frac{d}{d t}\left\|u_{t}\right\|^{2}(t)+v\left\|u_{t}\right\|_{V}^{2}(t) \\
& \quad+\left(v-\frac{18 L^{1 / 2}\|u\|^{1 / 4}(t)\left\|u_{t}\right\|^{3 / 4}(t)}{v^{3 / 4} \pi^{1 / 2}}\right)\left\|\nabla u_{t}\right\|^{2}(t)
\end{aligned}
$$

$\leq 0$.

Rewrite this in the form

$$
\begin{aligned}
& \frac{d}{d t}\left\|u_{t}\right\|^{2}(t)+v\left\|u_{t}\right\|_{V}^{2}(t) \\
& \quad+L^{1 / 2}\left(\frac{\left[\pi^{1 / 2} / L^{1 / 2}\right] v^{7 / 4}-18\left\|u_{0}\right\|^{1 / 4}\left\|u_{t}\right\|^{3 / 4}(t)}{\pi^{1 / 2} \nu^{3 / 4}}\right) \\
& \quad \cdot\left\|\nabla u_{t}\right\|^{2}(t) \leq 0 .
\end{aligned}
$$

Taking into account conditions (25) of Theorem 5, we get that

$$
\left[\frac{\pi^{1 / 2}}{L^{1 / 2}}\right] v^{7 / 4}-18\left\|u_{0}\right\|^{1 / 4}\left\|u_{t}\right\|^{3 / 4}(t)>0, \quad t>0 ;
$$

hence (44) reduces to the form

$$
\frac{d}{d t}\left\|u_{t}\right\|^{2}(t)+v\left\|u_{t}\right\|_{V}^{2}(t) \leq 0
$$

By the Steklov inequality,

$$
\frac{d}{d t}\left\|u_{t}\right\|^{2}(t)+\chi\left\|u_{t}\right\|^{2}(t) \leq 0
$$

We estimate

$$
\begin{aligned}
\left\|u_{t}\right\|(0) & \leq v\left\|\Delta u_{0}\right\|+\left\|u_{0}\right\|_{L^{4}(\Omega)}\left\|\nabla u_{0}\right\|_{L^{4}(\Omega)} \\
& \leq v\left\|\Delta u_{0}\right\|+C\left\|u_{0}\right\|_{H^{2}(\Omega) \cap V}^{2} \\
& \equiv C\left(\left\|u_{0}\right\|_{H^{2}(\Omega) \cap V}^{2}\right) .
\end{aligned}
$$

This and (46), (47) imply that $u_{t} \in L^{\infty}(0, \infty ; H) \cap$ $L^{2}(0, \infty ; V)$, and

$$
\left\|u_{t}\right\|^{2}(t) \leq C\left\|u_{0}\right\|_{H^{2}(\Omega) \cap V}^{2} e^{-\chi t} .
$$

Returning to (39), we get $u \in L^{\infty}(0, \infty ; V)$. This and (33), (49) prove validity of (27), (28), and consequently the existence part of Theorem 5.

Uniqueness of the Strong Solution. Let $u$ and $v$ be two strong solutions to (22) satisfying (23) and (27). Define $z=u-v$. Then for $\Phi \in V$ we have

$$
\left(z_{t}, \Phi\right)+(A z, \Phi)+b(u, z, \Phi)-b(z, z, \Phi)=0 .
$$

Taking $\Phi=z$, we come to the inequality

$$
\begin{aligned}
& \frac{d}{d t}\|z\|^{2}(t)+2 v\|z\|_{V}^{2}(t)=b(z, z, u)(t) \\
& \quad \leq 18\|u\|_{V}(t)\|z\|_{V}^{2}(t)
\end{aligned}
$$

that can be rewritten as

$$
\begin{aligned}
& \frac{d}{d t}\|z\|^{2}(t)+v\|z\|_{V}^{2}(t)+\left(\nu-18\|u\|_{V}(t)\right)\|z\|^{2}(t)_{V} \\
& \quad \leq 0 .
\end{aligned}
$$

Acting in the same manner as by the proof of Estimate II, we come to the inequality

$$
\begin{aligned}
& \frac{d}{d t}\|z\|^{2}(t)+v\|z\|_{V}^{2}(t) \\
& +L^{1 / 2}\left(\frac{\left[\pi^{1 / 2} / L^{1 / 2}\right] v^{7 / 4}-18\|u\|^{1 / 4}(t)\left\|u_{t}\right\|^{3 / 4}(t)}{\pi^{1 / 2} \nu^{3 / 4}}\right) \\
& \cdot\|z\|_{V}^{2}(t) \leq 0 .
\end{aligned}
$$


By conditions of Theorem 5,

$$
\frac{\left[\pi^{1 / 2} / L^{1 / 2}\right] \nu^{7 / 4}-18\|u\|^{1 / 4}(t)\left\|u_{t}\right\|^{3 / 4}(t)}{\pi^{1 / 2} \nu^{3 / 4}}>0
$$

for $t=0$. Taking into account Estimates (28) and using standard arguments, we get for all $t>0$

$$
\frac{\left[\pi^{1 / 2} / L^{1 / 2}\right] v^{7 / 4}-18\|u\|^{1 / 4}(t)\left\|u_{t}\right\|^{3 / 4}(t)}{\pi^{1 / 2} v^{3 / 4}}>0 .
$$

Hence, (53) becomes

$$
\frac{d}{d t}\|z\|^{2}(t) \leq 0
$$

This implies $z \equiv 0$ that proves uniqueness of the strong solution and completes the proof of Theorem 5 .

\section{More Regularity}

Consider the Poisson problem in a bounded domain $\Omega \in \mathbb{R}^{n}$ :

$$
\begin{aligned}
\Delta u & =f(x),(x) \in \Omega, \\
\left.u\right|_{\partial \Omega} & =0 .
\end{aligned}
$$

In [15] Theorem 11, p. 120-123, the following has been proved.

Lemma 6. Let

$$
\Omega_{\pi}=\left\{x=\left(x_{1}, \ldots, x_{n}\right), 0<x_{i}<\pi ; i=1, \ldots, n\right\}
$$

then the unique weak solution $u \in H_{0}^{1}(\Omega)$ of (57) satisfies the following inequality:

$$
\|u\|_{W^{2, p}\left(\Omega_{\pi}\right)} \leq C(\Omega)\|f\|_{L^{p}\left(\Omega_{\pi}\right)} .
$$

It is possible to generalize this result for a bounded parallelepiped in $\mathbb{R}^{4}$.

Theorem 7. The problem (57) posed in a parallelepiped $\Omega=$ $\left\{(x) \in \mathbb{R}^{4}, 0<x_{i}<L_{i} ; i=1,2,3,4\right\}$, where $f \in L^{p}(\Omega)$, $1<p \leq 2$, has a solution $u \in W^{2, p}(\Omega)$. Moreover,

$$
\|u\|_{W^{2, p}(\Omega)} \leq C_{\Omega}\|f\|_{L^{p}(\Omega)} .
$$

Returning to the original problem for the Navier-Stokes equations,

$$
\begin{aligned}
u_{t}-v \Delta u+\nabla p+(u \cdot \nabla) u=0 & \text { in } \Omega \times(0, t), \\
\nabla \cdot u=0 & \text { in } \Omega \times(0, t), \\
u=0 &
\end{aligned}
$$

$$
\text { in } \partial \Omega \times(0, t), t>0,
$$$$
u(x, 0)=u_{0}(x) \text { in } \Omega \text {, }
$$

where $u(x)$ is a vector function from $\mathbb{R}^{4}$ into $\mathbb{R}^{4}$ and $p$ is a real function from $\mathbb{R}^{4}$ into $\mathbb{R}$, and making use of Galerkin approximations, we establish the following result.
Theorem 8. Given $u_{0} \in H^{2}(\Omega) \cap V$ and a domain $\Omega$ satisfying (25), then problem (61) has a unique regular solution $(u, p)$ such that

$$
\begin{gathered}
u \in L^{\infty}\left(0, \infty ; V \cap W^{2,4 / 3}(\Omega)\right), \\
u_{t} \in L^{\infty}(0, \infty ; H) \cap L^{2}(0, \infty ; V), \\
\nabla p \in L^{\infty}\left(0, \infty ; L^{4 / 3}(\Omega)\right)
\end{gathered}
$$

which for all $\Phi(x) \in V$ satisfies the following integral identity:

$$
\int_{\Omega}\left\{u_{t}+v \Delta u+(u \cdot \nabla) u\right\} \Phi d x=0
$$

Moreover,

$$
\begin{aligned}
& \left\|u_{t}\right\|(t)+\|u\|(t)_{W^{2,4 / 3}(\Omega)}+\|\nabla p\|_{L^{4 / 3}(\Omega)}(t) \\
& \quad \leq C e^{-(1 / 2) \chi t}
\end{aligned}
$$

where $\chi=v \pi^{2} \sum_{i=1}^{4}\left(1 / L_{i}^{2}\right)$ and $C$ depends on $\left\|u_{0}\right\|_{H^{2}(\Omega)}$.

Proof (decay of $W^{2,4 / 3}(\Omega)$-norm). Taking into account that conditions of Theorem 8 and of Theorem 5 are the same, by Theorem 5, we have a unique strong solution of (61). Hence, to prove Theorem 8 , it is sufficient to establish that $\|u\|_{W^{2,4 / 3}(\Omega)}(t)$. First write (22) as

$$
\Delta u=f=\frac{1}{v}\left(u_{t}+\nabla p+(u \cdot \nabla) u\right) .
$$

We estimate

$$
\begin{aligned}
|b(u, u, v)|(t) & =|((u \cdot \nabla) u, v)(t)| \\
& \leq C\|u\|(t)_{L^{4}(\Omega)}\|u\|(t)_{V}\|v\|(t)_{L^{4}(\Omega)} \\
& \leq C\|u\|^{2}(t)_{V}\|v\|(t)_{L^{4}(\Omega)}
\end{aligned}
$$

Hence by (33), (49), (51),

$$
\|B u\|(t)_{L^{4 / 3}(\Omega)}=\|(u \cdot \nabla) u\|(t)_{L^{4 / 3}(\Omega)} \leq C e^{-(3 / 2) \chi t} .
$$

Returning to (23) and making use of (67), (49), we obtain

$$
\begin{aligned}
\|A u\|_{L^{4 / 3}(\Omega)}(t) & \leq\|B u\|_{L^{4 / 3}(\Omega)}(t)+\left\|u_{t}\right\|_{L^{2}(\Omega)}(t) \\
& \leq C e^{-(\chi / 2) t} .
\end{aligned}
$$

By the Theorem of de Rham (see [7, 18]), [9] Propositions 1.1, 1.2 , p. 10, one can check that there exists $\nabla p$ such that (see [9])

$$
-\nabla p=u_{t}+A u+B u
$$

and

$$
\begin{aligned}
\|\nabla p\|_{L^{4 / 3}(\Omega)}(t) \leq & \left\|u_{t}\right\|_{L^{2}(\Omega)}(t)+\|A u\|_{L^{4 / 3}(\Omega)}(t) \\
& +\|B u\|_{L^{4 / 3}(\Omega)}(t) \leq C e^{-(\chi / 2) t} .
\end{aligned}
$$


Returning to (65) and having $f \in L^{4 / 3}(\Omega)$, we obtain, due to Theorem 7,

$$
\begin{aligned}
\|u\|(t)_{W^{2,4 / 3}(\Omega)} \leq & \left\|u_{t}\right\|_{L^{2}(\Omega)}(t)+\|\nabla p\|_{L^{4 / 3}(\Omega)}(t) \\
& +\|(u \cdot \nabla) u\|_{L^{4 / 3}(\Omega)}(t) \leq C e^{-(\chi / 2) t} .
\end{aligned}
$$

By the Sobolev theorems,

$$
\|u\|_{L^{4}(\Omega)}(t) \leq C\|u\|_{W^{2,4 / 3}(\Omega)}(t) \leq C e^{-(\chi / 2) t} .
$$

The proof of Theorem 8 is complete.

Remark 9. It follows from (72) that $B u \in L^{4 / 3}$ and consequently, $f \in L^{4 / 3}(\Omega)$ and $u \in W^{2,4 / 3}(\Omega)$ in (60). This means that we cannot achieve better regularity then $u \in W^{2,4 / 3}(\Omega)$. In some sense, this is the superior regularity for the problem (61). It looks like $n=4$ is the critical case of the Navier-Stokes system.

Existence and Decay of a Strong Solution on an Unbounded $4 D$ Domain. Define an unbounded four-dimensional parallelepiped $D=\left\{x \in \mathbb{R}^{4} ; 0<x_{i}<L_{i}, i=1,2,3 ; 0<x_{4}<+\infty\right\}$ and let $L_{m}=\min \left(L_{1}, L_{2}, L_{3}\right)$.

Given $u_{0} \in H^{2}(D) \cap V$, consider in $D \times(0, t)$ the following problem:

$$
\begin{array}{rlrl}
u_{t}-v \Delta u+\nabla p+(u \cdot \nabla) u & =0 & & \text { in } D \times(0, t), \\
\nabla \cdot u & =0 & & \text { in } D \times(0, t), \\
u & =0 & & \\
& \text { on } \partial D \times(0, t), t>0, \\
\lim _{4} \longrightarrow \infty(x, t) \mid & =0, \quad t>0, \\
u(x, 0) & =u_{0}(x) \quad \text { in } D .
\end{array}
$$

Theorem 10. Given $u_{0} \in H^{2}(D) \cap V$ and $D$ such that

$$
\left(\frac{\pi}{L_{m}}\right)^{1 / 2} v^{7 / 4}-18\left\|u_{0}\right\|^{1 / 4}\left\|u_{t}\right\|^{3 / 4}(0)>0
$$

there exists a unique strong solution $u(x, t)$ to (73):

$$
\begin{aligned}
& u \in L^{\infty}(0, \infty ; V), \\
& u_{t} \in L^{\infty}(0, \infty ; H) \cap L^{2}(0, \infty ; V)
\end{aligned}
$$

such that for all $\Phi: \mathbb{R}^{4} \longrightarrow \mathbb{R}^{4} \in V,\left.\Phi\right|_{\partial D}=0$, tending sufficiently rapidly to 0 as $x_{4} \longrightarrow \infty$, it satisfies the following identity:

$$
\begin{array}{r}
\left(u_{t}, \Phi\right)(t)+v\left(\sum_{i=1}^{4} D_{i} u, D_{i} \Phi\right)(t)+b(u, \Phi, u)(t)=0 \\
t>0 .
\end{array}
$$

Moreover, the following inequalities hold:

$$
\begin{aligned}
\|u\|(t) & \leq\left\|u_{0}\right\| e^{-\theta t}, \\
\left\|u_{t}\right\|(t) & \leq\left\|u_{t}\right\|(0) e^{-(\theta / 2) t}, \\
\mid u \|(t)_{V} & \leq C\left\|u_{0}\right\|\left\|u_{t}\right\|(0) e^{-(3 / 4) \theta t},
\end{aligned}
$$

where $\theta=v \pi^{2} \sum_{i=1}^{3}\left(1 / L_{i}^{2}\right)$.
Proof. Obviously, the variational formulation of (73) is also (23). Repeating the proof of Theorem 5, we can prove the existence and uniqueness of the strong solution to problem (73). Using the Steklov inequalities with respect to variables $x_{1}, x_{2}, x_{3}$, we obtain

$$
\|\nabla u\|^{2} \geq \sum_{i=1}^{3} \frac{\pi^{2}}{L_{i}^{2}}\|u\|^{2}+\left\|D_{4} u\right\|^{2} .
$$

hence, (23) becomes

$$
\frac{d}{d t}\|u\|^{2}(t)+2 v \sum_{i=1}^{3} \frac{\pi^{2}}{L_{i}^{2}}\|u\|^{2}(t)+2 v\left\|D_{4} u\right\|^{2}(t) \leq 0 .
$$

This implies

$$
\|u\|^{2}(t) \leq\left\|u_{0}\right\|^{2} e^{-2 \theta t}
$$

Repeating the proof of Estimate II of Theorem 5, we find

$$
\left\|u_{t}\right\|^{2}(t) \leq\left\|u_{t}\right\|^{2}(0) e^{-\theta t}
$$

and

$$
\begin{aligned}
\|u\|_{V}^{2}(t) & \leq \frac{1}{v}\left\|u_{t}\right\|(t)\|u\|(t) \\
& \leq \frac{1}{v}\left\|u_{0}\right\|\left\|u_{t}\right\|(0) e^{-(3 / 2) \theta t} .
\end{aligned}
$$

Decay for Pressure. In order to obtain decay for $\|\nabla p\|_{L^{4 / 3}(D)}(t)$, we start with

$$
\|(u \cdot \nabla) u\|_{L^{4 / 3}(D)}(t)=\|B u\|_{\left(L^{4}(D)\right)^{\prime}}(t),
$$

where $\left(L^{4}(D)\right)^{\prime}$ is the dual to the space $L^{4}(D)$. Since

$$
A u=-u_{t}-B u
$$

then by (81) and (83),

$$
\|A u\|(t)_{L^{4 / 3}(\Omega)} \leq c_{1} e^{-(1 / 2) \theta t} .
$$

Moreover, by (69),

$$
\begin{aligned}
\|\nabla p\|_{L^{4 / 3}(D)}(t) \leq & \left\|u_{t}\right\|_{L^{2}(D)}(t)+\|A u\|_{L^{4 / 3}(D)}(t) \\
& +\|B u\|_{L^{4 / 3}(D)}(t) \leq c_{2} e^{-(1 / 2) \theta t} .
\end{aligned}
$$

Jointly (80), (81), and (86) prove Theorem 10.

Conclusions. In our work, we tried to respond to some questions posed by J. Leray [1], namely, regularity of global solutions of the Navier-Stokes equations and their decay. Therefore, our results can be divided into two parts: the first one concerns decay of global regular solutions of the 4D NavierStokes equations posed on bounded $4 \mathrm{D}$ parallelepipeds. It is known that there exist global regular solutions for the $2 \mathrm{D}$ Navier-Stokes equations posed on smooth bounded domains $[4,6,8,9]$, but regularity in nonsmooth (Lipschitz) domains is not obvious. For bounded $4 \mathrm{D}$ parallelepipeds, we have 
established the existence of a unique global regular solution which decays exponentially as $t \longrightarrow+\infty$ provided that initial data satisfies (25). We demonstrated that the decay rate is different for different norms; see (77), where $\theta$ is defined by the geometrical characteristics of a domain $\Omega$.

The second part of our work concerns decay of solutions for the 4D Navier-Stokes equations posed on an unbounded parallelepiped. In existing publications $[3,4,6,9]$, the decay rate of $\|u\|_{L^{2}(\Omega)}(t)$ is controlled by the first eigenvalue of the operator $A=-P \Delta$, where $P$ is the projection operator on a solenoidal subspace of $L^{2}(\Omega)$. It is clear that this approach does not work in unbounded domains.

On the other hand, our approach based on the Steklov inequalities allowed us to estimate the decay rate of a strong solution for the $4 \mathrm{D}$ Navier-Stokes equations posed on an unbounded 4D parallelepiped.

We must emphasize that this estimate is the first one which gives an explicit value of the decay rate for unbounded $4 \mathrm{D}$ domains. Results established in our work can be used in constructing of numerical schemes for solving initialboundary value problems for the Navier-Stokes equations appearing in Mechanics of viscous liquid. From the physical point of view, decay estimates show that the decay rate of perturbations of solutions caused by the initial data is bigger for bigger values of viscosity $v$ and smaller sizes of $4 \mathrm{D}$ parallelepipeds.

My interest for the 4D Navier-Stokes equations is purely mathematical and, on my opinion, can not be extended to higher dimensions beyond 4. I must also note that there are publications on the existence of weak solutions for 4D NavierStokes equations [7], [9] p.189-197.

\section{Data Availability}

The data used to support the findings of this study are included within the article.

\section{Conflicts of Interest}

The author declares that there are no conflicts of interest regarding the publication of this paper.

\section{Acknowledgments}

This research has been supported by Fundação Araucaria, Parana, Brazil, Convenio No. 307/2015, Protocolo No. 45.703.

\section{References}

[1] J. Leray, "Sur le mouvement d'un liquide visqueux emplissant l'espace," Acta Mathematica, vol. 63, no. 1, pp. 193-248, 1934 (French).

[2] R. M. Brown, P. A. Perry, and Z. Shen, "On the dimension of the attractor for the non-homogeneous Navier-Stokes equations in non-smooth domains," Indiana University Mathematics Journal, vol. 49, no. 1, pp. 81-112, 2000.

[3] D. E. Edmunds, "Asyptotic behavior of solutions of the NavierStokes equations," Archive for Rational Mechanics and Analysis, vol. 22, pp. 15-21, 1966.
[4] C. Foias and G. Prodi, "Sur le comportement global des solutions non-stationairos des equations de Navier-Stokes en dimension 2," Rendiconti del Seminario Matematico della Università di Padova. The Mathematical Journal of the University of Padova, vol. 39, pp. 1-34, 1967.

[5] C. Foias and J.-C. Saut, "Asymptotic Behavior, as $t \longrightarrow \infty$ of Solutions of Navier-Stokes Equations and Nonlinear Spectral Manifolds," Indiana University Mathematics Journal, vol. 33, no. 3, pp. 459-477, 1984.

[6] O. A. Ladyzhenskaya, The Mathematical Theory of Viscous Incompressible Flow, Gordon and Breach, New York, NY, USA, 2nd edition, 1969.

[7] J. L. Lions, Quelques Méthodes de Résolution des Problèmes aux Limites Non Linéaires, Dunod, Paris, Farnce, 1969.

[8] G. Prodi, "Qualche risultato riguardo alle equazioni di NavierStokes nel caso bidimensional," The Mathematical Journal of the University of Padova, vol. 30, pp. 1-15, 1960.

[9] R. Temam, Navier-Stokes Equations. Theory and Numerical Analysis, North-Holland Publishing, Amsterdam, The Netherlands, 1979.

[10] G. Raugel and G. R. Sell, "Navier-Stokes equations on thin 3D domains. I. Global attractors and global regularity of solutions," Journal of the American Mathematical Society, vol. 6, no. 3, pp. 503-568, 1993.

[11] R. Temam and M. Ziane, "Navier-Stokes equations in threedimensional thin domains with various boundary conditions," Advances in Differential Equations, vol. 1, no. 4, pp. 499-546, 1996.

[12] P. Deuring and W. von Wahl, "Strong solutions of the NavierStokes system in Lipschitz bounded domains," Mathematische Nachrichten, vol. 171, pp. 111-148, 1995.

[13] J. Malek, M. Padula, and M. Ruzicka, "A note on derivative estimates for a Hopf solution to the Navier-STOkes system in a three-dimensional cube," in Navier-Stokes Equations and Related Nonlinear Problems, A. Sequeira, Ed., pp. 141-146, Plenum, UK, 1995.

[14] A. A. Kiselev and O. A. Ladyzhenskaya, "On the existence and uniqueness of the solution of the nonstationary problem for a viscous, incompressible fluid," Izvestiya Akademii Nauk SSSR Seriya Matematicheskaya, vol. 21, pp. 655-680, 1957.

[15] A. I. Koshelev, "A priori estimates in Lp and generalized solutions of elliptic equations and systems," American Mathematical Society Translations, vol. 20, no. 2, pp. 105-171, 1962.

[16] A. V. Steklov, "The problem of cooling of an heterogeneous rigid rod," Communs. Kharkov Math. Soc., Ser, vol. 2, no. 5, pp. 136$181,1896$.

[17] O. A. Ladyzhenskaya, The Boundary Value Problems of Mathematical Physics, vol. 49 of Applied Mathematical Sciences, Springer, New York, NY, USA, 1985.

[18] G. de Rham, Variétés différentiables, Herman, Paris, France, 1960. 


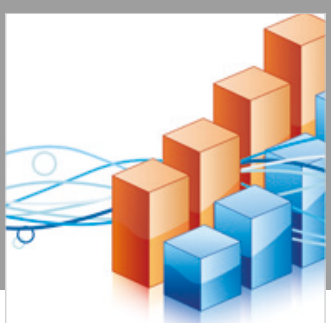

Advances in

Operations Research

\section{-n-m}
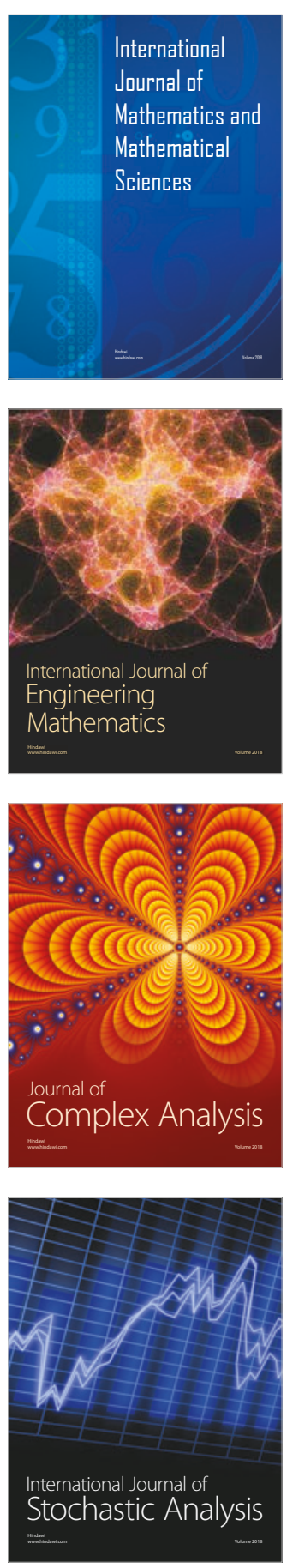
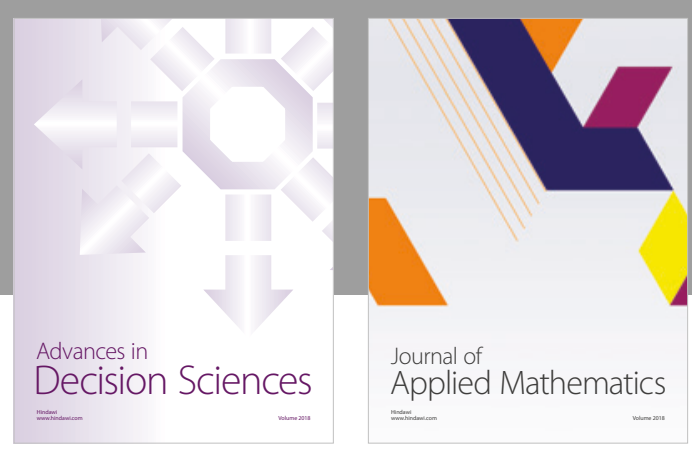

Journal of

Applied Mathematics
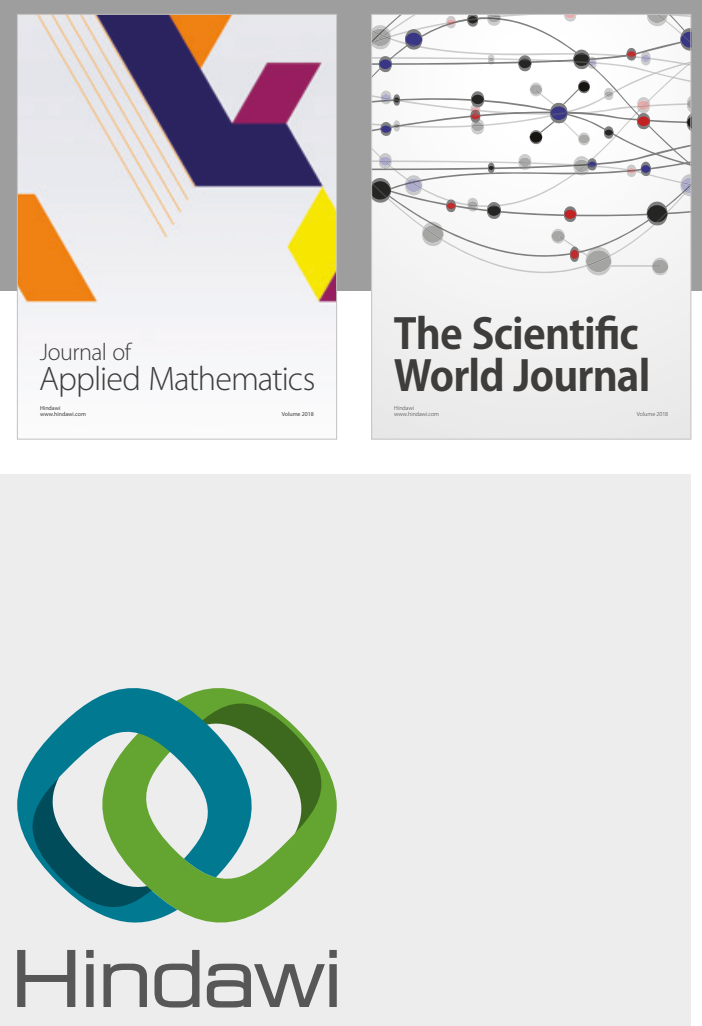

Submit your manuscripts at

www.hindawi.com

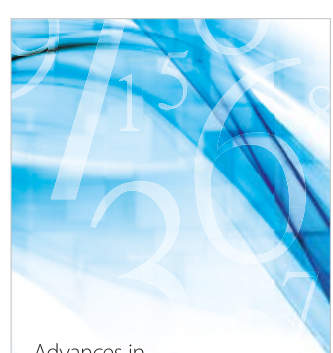

Advances in
Numerical Analysis
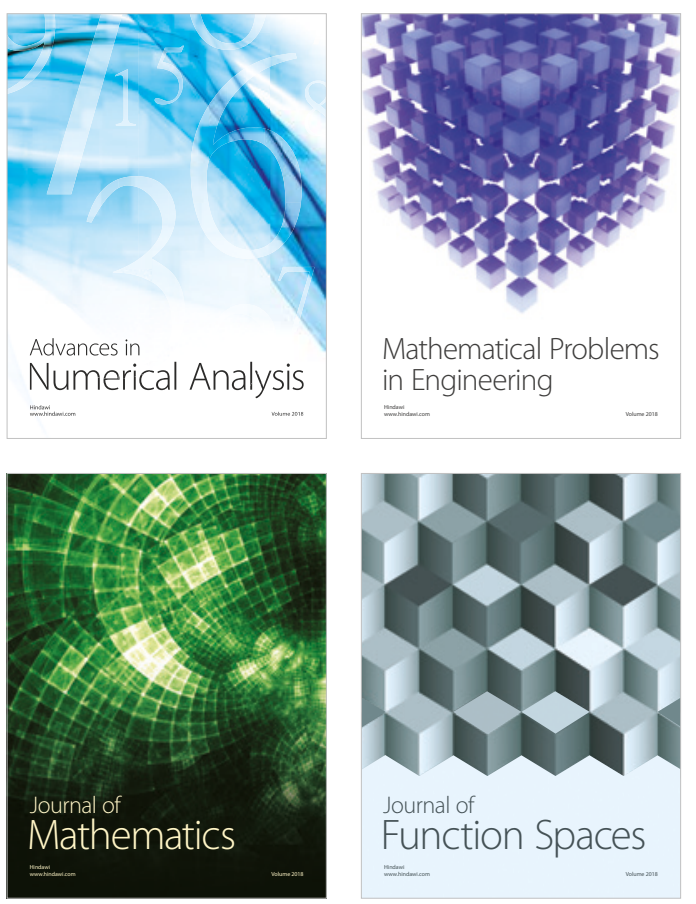

Mathematical Problems in Engineering

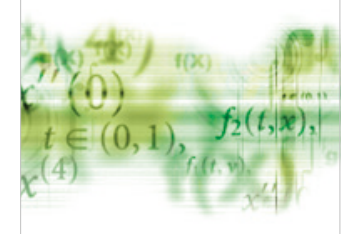

International Journal of

Differential Equations

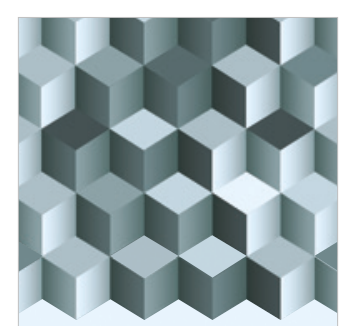

Journal of

Function Spaces

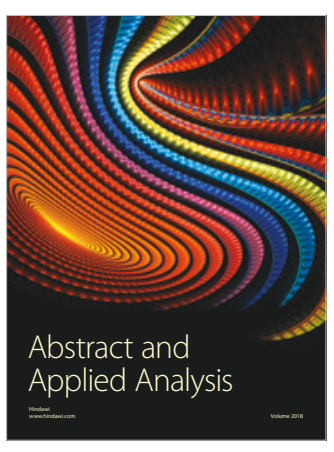

The Scientific

World Journal

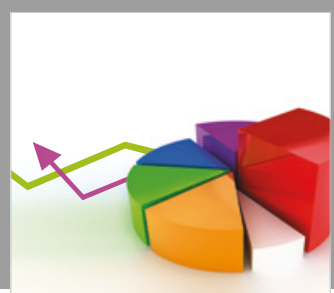

Journal of

Probability and Statistics
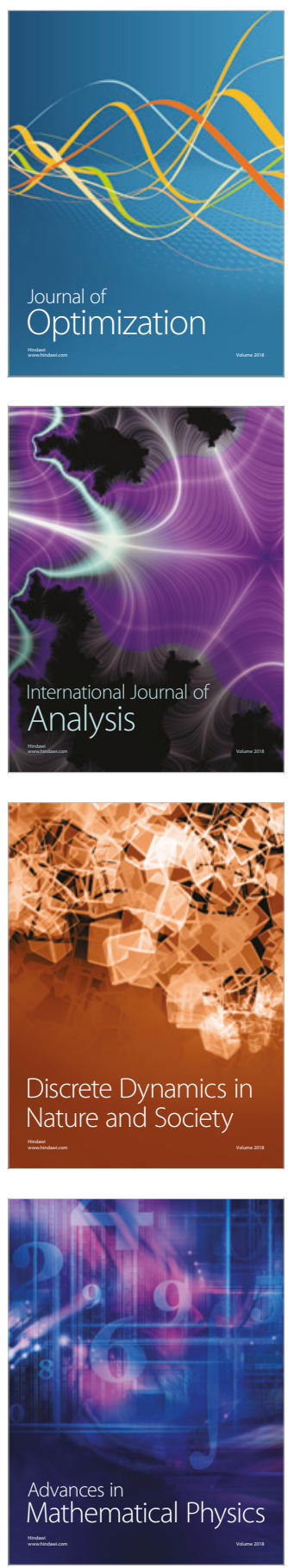